\title{
Arborescences
}

Revue d'études françaises

\section{En contre-jour : la représentation évanescente de l'écrivain dans le roman québécois contemporain}

\section{David Bélanger}

Numéro 6, septembre 2016

Polyphonies : voix et valeurs du discours littéraire

URI : https://id.erudit.org/iderudit/1037504ar

DOI : https://doi.org/10.7202/1037504ar

Aller au sommaire du numéro

Éditeur(s)

Département d'études françaises, Université de Toronto

ISSN

1925-5357 (numérique)

Découvrir la revue

Citer cet article

Bélanger, D. (2016). En contre-jour : la représentation évanescente de l'écrivain dans le roman québécois contemporain. Arborescences, (6), 54-71.

https://doi.org/10.7202/1037504ar

\section{Résumé de l'article}

La représentation de l'écrivain dans la littérature québécoise s'inscrit dans une histoire d'émancipation, d'inadéquations et de résistances. Depuis les premiers travaux d'André Belleau, cette représentation a évolué et, dans les années 2000 , se profile une figure de romancier à la fois plus confiante et plus évanescente que celle des années 1960-1970. L'écrivain, en s'affichant dans sa fiction, montre les attaques répétées faites par la théorie et par la société à son autorité créatrice. C'est ce que nous proposons d'observer, en nous reportant à Une estafette chez Artaud de Nicolas Tremblay et à Pleurer comme dans les films de Guillaume Corbeil. En abordant diverses figures auctoriales (Bartleby, l'anti-écrivain, l'écrivain frauduleux, etc.), nous serons amené à penser, par le fait même, le statut de la littérature dans la production littéraire québécoise contemporaine.
Tous droits réservés @ Département d'études françaises, Université de Toronto, 2016 document est protégé par la loi sur le droit d'auteur. L’utilisation des services d'Érudit (y compris la reproduction) est assujettie à sa politique d'utilisation que vous pouvez consulter en ligne. 


\section{SOMMAIRE}

Francis Langevin et Raphaël Baroni.

01 University of British Columbia et Université de Lausanne Introduction

Alain Rabatel. Université de Lyon 1

13 L'énonciation problématisante : en dialogue avec

Le Royaume d'Emmanuel Carrère

Émilie Goin. Université du Luxembourg

39 Analyse d'un discours d'action collective mis en récit.

L'Anarchie dans Les Cloches de Bâle d'Aragon

David Bélanger. Université du Québec à Montréal

54 En contre-jour : la représentation évanescente de l'écrivain dans le roman québécois contemporain

Raphaël Baroni. Université de Lausanne

72 Comment débusquer la voix d'un auteur dans sa fiction?

Une étude de quelques provocations de Michel Houellebecq

Liesbeth Korthals Altes. Rijksuniversiteit Groningen

94 Actes de cadrage, narratologie et herméneutique - à propos de I'indétermination énonciative dans Sujet Angot de Christine Angot

121

Marion Sauvaire et Érick Falardeau. Université Laval

Susciter le moment critique. De l'investissement de ressources axiologiques

Frank Wagner. Université Rennes 2

148 Quand le narrateur boit(e)... (Réflexions sur le narrateur non fiable et/ou indigne de confiance) 


\title{
En contre-jour : la représentation évanescente de l'écrivain dans le roman québécois contemporain
}

\author{
David Bélanger. Université du Québec à Montréal
}

\section{Résumé}

La représentation de l'écrivain dans la littérature québécoise s'inscrit dans une histoire d'émancipation, d'inadéquations et de résistances. Depuis les premiers travaux d'André Belleau, cette représentation a évolué et, dans les années 2000, se profile une figure de romancier à la fois plus confiante et plus évanescente que celle des années 1960-1970. L'écrivain, en s'affichant dans sa fiction, montre les attaques répétées faites par la théorie et par la société à son autorité créatrice. C'est ce que nous proposons d'observer, en nous reportant à Une estafette chez Artaud de Nicolas Tremblay et à Pleurer comme dans les films de Guillaume Corbeil. En abordant diverses figures auctoriales (Bartleby, l'antiécrivain, l'écrivain frauduleux, etc.), nous serons amené à penser, par le fait même, le statut de la littérature dans la production littéraire québécoise contemporaine.

Au cours du vingtième siècle, des théoriciens de diverses allégeances ont tour à tour annoncé la mort du personnage, de l'intrigue, des descriptions, et même de l'auteur dans le roman moderne. Plus récemment, les prophètes des nouvelles technologies ont prédit la disparition prochaine du livre en tant qu'objet. Un roman, de nos jours, devrait donc être une chose dépourvue de personnages, d'intrigue, de descriptions, d'auteur et de support matériel. C'est dire à quel point vous venez de vous faire filouter si vous avez déboursé vingt dollars pour celui-ci. (François Blais (2007: 9)

Entrons dans le vif du sujet, aussi bien dire par un diagnostic : l'auteur fictif que présente la littérature québécoise contemporaine tend à disparaître, son texte semble assourdir sa voix, l'œuvre éparpille ses énoncés. L'incipit de Nous autres ça compte pas, préface étrange prise en charge par l'auteur fictif du récit, en propose (voir l'exergue) une éloquente démonstration. Cet incipit renvoie aussitôt le lecteur au paradoxe pragmatique de la théorie : en effet, l'auteur ne peut pas être mort puisque je tiens ce livre entre mes mains et que celui-ci contient des mots assemblés en phrases puis en intrigue. Tout le livre de Blais est d'ailleurs un rappel de l'exercice pragmatique qu'est la rédaction du roman. L'écrivain pianote sur son ordinateur un récit (métadiégétique, 
selon les termes de Genette ${ }^{1}$ ), devant un témoin, un vieil homme étrange fasciné par l'activité d'écriture. Les pratiques prosaïques de l'écriture sont présentées contre le lyrisme de l'écrivain à l'œuvre: l'auteur, nous dit le témoin, copie-colle des morceaux d'autres documents, recyclant ses anciens écrits, son rythme varie selon les passages et il vérifie régulièrement le nombre de mots écrits comme on mesure sa productivité. Le spectateur de l'écriture va jusqu'à s'exclamer : "En fait, pour dire les choses rondement : vous voir écrire est l'un des spectacles les plus ennuyants auxquels j'ai assisté de ma vie » (35). L'ennui de l'écriture se répercutera sur l'ennui du récit métadiégétique, de l'avis du même spectateur : "Mais nonobstant la question de la forme, peuton savoir où tout cela nous mène, au juste ?" (175) L'auteur, agacé par ce lecteur, choisit de le faire disparaître de son texte : "Il n'est pas mauvais, une fois de temps en temps, de leur rappeler qui est le patron" (176).

L'écrivain, dans ce roman de François Blais, occupe le rôle central. Sa figure de scripteur assure l'unité de l'énonciation et l'unité thématique, et si ses personnages semblent être faits de papier, si l'intrigue est sinon absente, du moins mince, c'est que l'auteur est tout dans le procès de l'œuvre, sa voix est garante du livre. Cette omnipotence auctoriale fait en sorte que Nous autres ça compte pas jure quelque peu avec la représentation du romancier de son époque, dans laquelle l'auteur tend plutôt à disparaître. En effet, on constate généralement que l'auteur se trouve aux prises avec une instance étrangère qui menace son autonomie, son autorité, son auctorialité, et le geste inhabituel du roman de Blais, celui de rejeter l'autre, s'il est original, ne se déroule que dans l'espace d'une lutte théorique et pragmatique balisée par nombre de critiques. Cet espace de lutte, il faut le rappeler, n'est pas l'unique conséquence d'une "mort de l'auteur" comme l'indique l'incipit du roman, elle est aussi la conséquence - postmoderne ? - d'une conception ouverte des discours, où chaque parole est tressée d'interdiscours, embrouillée dans un dialogisme, des présupposés sociaux, une intertextualité totale qui aurait raison de la parole authentique. Plus encore, l'analyse pragmatique et sociologique du texte le confine au sein d'enjeux institutionnels, où la légitimité et la consécration sont affaire de marché symbolique ; le texte est alors pris en charge par la voix de l'éditeur, la voix du critique, la voix de l'universitaire, la voix anonyme des lecteurs, le texte trouve son identité dans cette multiplicité d'énonciateurs. En conséquence de quoi, et c'est l'hypothèse du présent article, on observe plus que jamais des romanciers fictifs qui perdent ou bien la parole ou bien leur autorité sur celle-ci. Rares sont ceux qui repousseront du texte l'autre envahissant pour " rappeler qui est le patron "; ils devront trouver un nouvel équilibre pour exister.

Charline Pluvinet visitait déjà une telle hypothèse dans son Fictions en quête d'auteur. Se penchant sur les fictions contemporaines, européennes et

${ }^{1}$ Il s’agit du « discours emboîté » ou « récit dans le récit » (Genette 1972 : 67-273). 
anglo-saxonnes, elle observait cette "disparition » de l'auteur fictif au sein de son texte, mais postulait en même temps que ce jeu antithétique de la représentation du romancier dans la fiction se faisait " non pour y disparaître mais pour redéfinir les conditions d'un maintien de l'auteur dans le champ littéraire" (2012: 12). Cette piste de la "reconquête» de l'autorité de l'auteur, autorité qui aurait été perdue dans la culture récente, trouve une résonance particulière dans le Québec contemporain. On sait quelle fortune ont connu les recherches sur le romancier fictif au Québec: alors qu'en France le structuralisme travaillait à liquider le "sujet créateur ", André Belleau travaillait à une thèse sur la représentation du romancier, thèse riche d'hypothèses et d'intuitions. Entre autres, soutenait Belleau, avant 1960, le romancier représenté était incomplet, son statut socioculturel ne se mariait jamais complètement à sa tâche d'écriture. Ce ne sera qu'avec l'ère du soupçon, autour de 1965, que le roman du romancier fictif

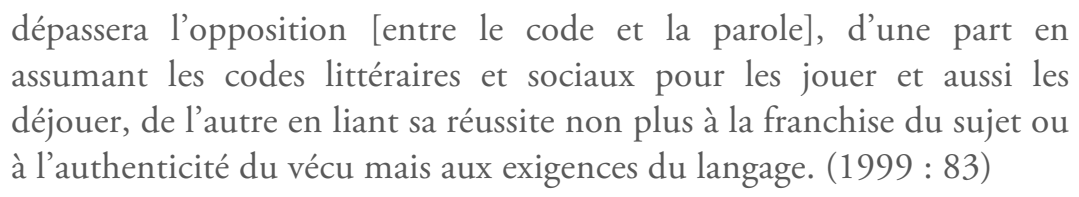

Cette hypothèse, quelque peu idyllique, résistera difficilement au temps : le thème de l'impossible complétude du romancier québécois - toujours représenté dans son incapacité à écrire, à exprimer son temps, son histoire, le code littéraire - revient sur différents tons dans les travaux de Gilles Marcotte (1976), Michel Biron (2000) ou Roseline Tremblay (2004). Mais, comme si elle témoignait d'un saut dans la modernité, la représentation contemporaine du romancier fictif ne semble plus la représentation d'une incapacité, mais plutôt d'une négativité. Pour mieux dire : la parole ne résiste plus à l'écrivain, c'est l'écrivain qui y résiste. Il n'est plus dépassé par le code, il veut le dépasser. Apparaîtrait alors ce qu'on pourrait nommer "l'anti-écrivain ", figure auctoriale gênée par son statut, désirant travailler la littérature par le maquis, clandestinement, en contre-jour. C'est cette figure que j'entends observer, par l'étude de deux manifestations distinctes. Je m'attarderai d'abord à l'écrivain négatif, le " bartleby ", notamment théorisé par Patrick Tillard ; il est le refus radical de la parole tel que le présente Une estafette chez Artaud de Nicolas Tremblay (2011). Je me pencherai ensuite sur l'écrivain frauduleux, auquel n'appartient pas la parole littéraire qu'il exhibe comme sienne ; on trouvera un exemple éclairant dans Pleurer comme dans les films de Guillaume Corbeil (2009). Ce seront là les deux temps d'analyse de cet article, qui vise à saisir les modalités de cette négativité : comment l'écrivain fictif peut-il batailler avec ces voix de l'autre - critiques, institutionnelles, sociales - qui sapent son discours ? demanderai-je. En fin de parcours, il n'est pas interdit que le statut de la littérature dans les sociétés représentées constitue, comme lieu d'énonciation et comme discours constituant, la pierre 
d'achoppement, le trou noir de toutes ces négativités.

\section{L'écrivain négatif : je n'écris pas}

Le bartleby, écrivain négatif issu du récit d'Hermann Melville, est cet auteur qui "apparaît pour disparaître. C'est là la condition de son existence. Son implication se poursuit sans relâche dans rien d'autre que son silence et sa disparition" (Tillard: 131). L'écrivain négatif n'écrit pas, ou du moins, il reste hors du circuit de l'édition, car écrire, c'est se compromettre : "Se trahir pour exister est peut-être, en effet, le lot de la plupart des écrivains qui maîtrisent l'art du compromis afin d'être publiés" (Tillard: 153). Refuser cette trahison de la publication vise à préserver une sorte d'idéal de la littérature et suppose, en même temps, la déréliction de l'art littéraire, dont les fondements institutionnels seraient pourris. On le rencontre de façon saisissante dans les romans de Jean-Philippe Martel et de Marie-Christine Lemieux-Couture : les auteurs-narrateurs n'ont de cesse de refuser le roman qu'ils écrivent, de le désamorcer, de le nier comme parole et comme œuvre². Les discours crépusculaires de la littérature, en crescendo depuis les années 1980, ne sont pas étrangers à cette rhétorique du bartleby - une rhétorique de silence, pour l'essentiel. En ce sens, Henri Raczymow dans La mort du grand écrivain souligne la perte de prestige de la littérature corrélative à la perte de prestige du statut d'auteur : "Publier un livre aujourd'hui, écritil, est chose désespérément banale» (13). De cette banalité du littéraire, confondu de plus en plus avec les énoncés communs - et même, dans la théorie, avec le reste du "discours social»-, nait donc la révolte silencieuse du bartleby. Patrick Tillard explicite cette entreprise négative :

L'œil critique des bartlebys ne perçoit dans les œuvres que l'amère production éthérée d'une mise en scène scripturale, la reconduction du rôle du scripteur qui prend conscience de son ombre. Beaucoup plus que cela, le silence et les refus des écrivains négatifs sont à la fois l'observation d'une certaine quantité de vide atteint par la littérature dans son environnement social et surtout la soustraction à ce vide. (Tillard : 167)

\footnotetext{
${ }^{2}$ Retenons la fin du premier chapitre de Comme des sentinelles de Martel : "Je ne pouvais m'empêcher de tout confondre, les mots et les gens, leurs histoires et la mienne et, surtout, je me demande ce qui avait bien pu se produire pour que je me retrouve étendu dans ces draps maculés, seul, [...] alors que j'aurais pu me prélasser sur une terrasse, revenir de voyage, faire l'amour, renifler de la cocaïne ou écrire un roman " (10). Ou encore, les références soutenues, dans Toutes mes solitudes! de Lemieux-Couture au non-statut littéraire de son journal intime - lequel prend les allures, à plusieurs moments, d'un roman : "Moi ? Pas écrivaine du tout ! Je prosaille un peu, voilà ! Je réflexionne un tantinet, rien de poétique, je l'assure. L'écriture ne fait pas l'écrivain, tout est dans le style " (117).
} 
L'acte de soustraction de l'écrivain négatif serait à la fois un constat du vide littéraire et un dépassement de celui-ci. La figure de l'estafette, Nicolas Tremblay, dans Une estafette chez Artaud s'inscrit précisément dans ce topos.

Ce roman nous présente un écrivain, Nicolas Tremblay. Un écrivain qui serait mort en 2015 sans avoir publié quoi que ce soit :

Ses nombreux écrits, restés [...] inédits jusqu'à la fin de ses jours, prouvent néanmoins ses prétentions littéraires. [...] L'estafette ne souhaitait pas l'ultime consécration, devenue usuelle à son époque: c'est-à-dire voir son nom sur une couverture. Il s'échinait au contraire à effacer ses traces, à disparaître, à rester le seul dépositaire de sa parole qu'il préservait jalousement. [...] Car on publiait, de son vivant, trop de livres, la plupart médiocres, qui venaient alimenter une production industrielle gloutonne. (Une estafette chez Artaud [EA] : 83)

Cet écrivain en négatif ne constitue pas qu'une réaction à la "production industrielle gloutonne ": Nicolas est décrit dans son mépris total du système et même de l'institution, de cette organisation de valeurs autonome que Pierre Bourdieu nomme le champ littéraire. D'ailleurs, ce sont les termes de Bourdieu qu'on peut en partie reconnaître dans le rejet de Nicolas du champ de masse et du champ restreint :

Chez l'estafette, il y avait un dégoût profond pour la culture de masse et les diktats qu'elle impose [...]. Cependant, au contraire $\mathrm{d}$ [es autres artistes et intellectuels], il ne le faisait pas par snobisme ou par jalousie, car il abhorrait tout autant les universitaires [...] et leurs productions hermétiques que les cénacles d'auteurs embourgeoisés et subventionnés par l'État [.][...] L'accès à [ces] œuvres [hermétiques] restait ainsi fermé à ceux qui ne connaissaient pas le discours qui les légitimait. (EA : 147148)

Les diktats du champ de masse, comme contraintes outrageantes, sont rapprochés de la production restreinte, qui ne se développe qu'en vase clos, par ceux qui autorisent, dans le même mouvement, cette production ${ }^{3}$. En s'attaquant à tout ce système, il ne reste effectivement à l'auteur que le silence.

L'intérêt d'Une estafette chez Artaud ne réside évidemment pas dans

\footnotetext{
${ }^{3}$ On reconnaît ici la définition de l'artiste du champ autonome de Bourdieu, en effet: l'artiste autonome est cette "catégorie socialement distincte d'artistes ou d'intellectuels professionnels, de plus en plus enclins à ne connaître d'autres règles que celles de la tradition proprement intellectuelle ou artistique qu'ils ont reçue de leurs prédécesseurs et qui leur ont fourni un point de départ, ou un point de rupture, et de plus en plus en mesure de libérer leur production et leurs produits de toute servitude externe [...]" ("Le marché des biens symboliques » (1971: 51)).
} 
cette idéologie au premier degré, notamment parce que ce premier degré s'avère impossible: le texte que nous lisons existe. L'affabulation se révèle ainsi en tant qu'affabulation, le bartleby Nicolas Tremblay n'est bartleby que dans la fiction ; la réalité pragmatique du texte confond la théorie fictive du propos. En quatrième de couverture, tout est déjà limpide: "[Nicolas Tremblay] a publié en 2010, chez Lévesque éditeur, un recueil de nouvelles intitulé L'esprit en boîte. Une estafette chez Artaud est son premier roman ". Le paradoxe parait autrement productif: Nicolas Tremblay a écrit une fiction dans laquelle Nicolas Tremblay n'a jamais écrit de fiction. Pour ce faire, l'origine de la fiction doit être problématisée, ce que fait d'entrée de jeu une sorte de préface signée Jean-Nicolas, arrière-petit-fils de Nicolas Tremblay. Ce préfacier confie avoir trouvé, dans des circonstances énigmatiques, le manuscrit que nous lisons :

Récemment, je tombai, de retour de la polyvalente, en entrant dans ma chambre, sur un document assez volumineux, assemblé par une pince, qui trônait, bien en évidence, sur mon lit. [...] La première page porte un titre: Une estafette chez Artaud, et un sous-titre: Autogenèse littéraire. Il n'y a pas de nom d'auteur. J'ai pensé d'abord que c'était Nicolas, par un leurre dont il a le secret. Après avoir lu rapidement les premières pages, j'ai compris que cela pouvait être ma mère, Josephte. Écrit à la troisième personne, le roman raconte la vie de mon ancêtre, qui en est donc le personnage principal. Quant à savoir si Josephte en est bien l'auteure, j'en aurai une meilleure idée quand [...] j'en ferai la lecture, si seulement l'envie m'en prend, car, loin de la maison, je commencerai peut-être à renier définitivement mes racines, à oublier ma mère, mon père, mon patronyme et mon ancêtre. (EA : 33)

Notons pour l'instant deux choses : Jean-Nicolas est la création, d'un point de vue biologique, de Nicolas Tremblay. Il est une conséquence de son existence individuelle. Mais en tant que création, Jean-Nicolas menace de "renier " ses racines, de refuser son patronyme, ses ancêtres. De même, le manuscrit que nous nous apprêtons à lire, Une estafette chez Artaud, serait sans origine claire, c'est-à-dire que la fiction renierait, dans un geste inaugural révélateur, l'origine évidente - pragmatique et donc auctoriale - du texte. Le roman s'ouvre alors, porté par une narration impersonnelle, analytique et froide, une narration qui accueille toute la polyphonie des discours de la littérature: l'éditeur, par des extraits de ses mémoires, prend la parole, le critique aussi se prononce, Nicolas Tremblay lui-même peut être lu, grâce à son abondante correspondance sans destinataire ou à ses notes de cours. De façon indirecte, le discours des savants littéraires - les intertextes foisonnent - transparaît, mais aussi celui de "la plèbe vulgaire et ignorante" (EA : 134) opposée au monde des livres et représentée par la famille de Nicolas Tremblay. À leur manière, tous ces discours rejouent la négativité de la littérature. L'éditeur des éditions ZYX, Gaétan Lévesque, serait, par exemple, frappé par une sorte de 
malédiction dont il rendrait compte dans ses mémoires :

La spirale qui a emporté mon entreprise s'est nettement intensifiée après la visite de Nicolas Tremblay dans mes bureaux. [...] Au moment où je couche mes mémoires sur papier, j'ai l'intime conviction que la littérature - pour laquelle je me suis pourtant sacrifié - s'est retournée finalement contre moi. (EA : 62)

Ce que révèle ici l'éditeur, c'est bien la défaite des deux vecteurs constitutifs du littéraire, à savoir son pôle économique et industriel - la faillite de l'édition - et son pôle symbolique - la littérature, comme discours, se révolte. Les mémoires se poursuivent jusqu'en 2020 alors que Gaétan Lévesque trace un véritable paysage apocalyptique: les auteurs tombent comme des mouches, et leur mort en nombre semble annoncer la fin de la littérature. De la même manière, un critique journalistique, ayant assisté à une sorte de performance théâtrale de Nicolas Tremblay, aurait écrit: "C'est à se demander quelle mouche a piqué Nicolas Tremblay de croire qu'imiter Artaud aujourd'hui révolutionnerait le théâtre québécois. Son jeu n'est pas avant-gardiste, c'est du réchauffé » (EA : 46). En guise de réponse, la narration rappelle les préceptes de Nicolas, regrettant que, "d'objet collectif qu'elle était auparavant, la littérature [soit] devenue le moyen privilégié de l'expression des individus" (EA : 47). La doxa journalistique, la critique culturelle comme lieu de légitimation, est alors présentée dans son échec: incapable de défendre l'idéal symbolique de la littérature, le critique ne sait en parler que sous la forme d'un objet narcissique, fait pour l'usage et la gloire personnels. Si à cela on ajoute la parole du père, véritable voix du peuple, voix extérieure à la communauté interprétative des littéraires, le portrait négatif de la littérature semble complet :

Comme nombre de ses contemporains, [Marc Tremblay] voyait dans la fiction littéraire une simple source de distraction sans réelles conséquences. [...] S’il n'avait pas vécu la Révolution tranquille, il aurait certes jugé la littérature sur le plan moral, comme autrefois l'Église catholique, en l'accusant d'encourager le vice. Selon sa perspective désormais moderne et laïque, cela ne valait même pas la peine de lui prêter une quelconque attention. (EA : 132-133)

Sans pouvoir, la littérature ne vaut même pas la peine qu'on s'y attarde, ne serait-ce que pour la faire taire. Réservée aux happy few, à cette "drôle d'engeance passéiste qui, au XXe siècle finissant, prêchait encore dans le désert, à l'écart de la vie courante" (EA : 134), elle n'existe pas, tout simplement, pour l'ensemble de la société.

Reprenons du début: Une estafette chez Artaud de Nicolas Tremblay raconte, dans un style impersonnel, la non-écriture de Nicolas Tremblay, son 
statut négatif dans le milieu littéraire de son époque et, ce faisant, montre la déchéance de la littérature, par les paroles de l'éditeur, de la critique et du père - aussi bien dire du peuple -, signifiant toutes, d'une façon ou d'une autre, l'échec d'un idéal littéraire. Mais cette polyphonie ne semble permise qu'en raison du reniement premier du livre, soit son rejet pragmatique de l'auteur. Reste, par conséquent, un paradoxe : pour dire le projet négatif de Nicolas Tremblay, il faut produire un texte et en cela annuler, en partie, la négativité du projet. Plus encore, si ce texte s'attaque aux fondements de la littérature, il ne peut exister, comme énoncé pragmatique, que dans le champ de cette littérature - malgré la disparition de l'éditeur qui rend la publication possible, malgré l'incompétence du lecteur critique, malgré l'indifférence de la masse. C'est ce paradoxe qu'il faut maintenant observer.

Le statut impersonnel et anonyme du texte Une estafette chez Artaud, tel qu'il se donne à lire dans la préface de Jean-Nicolas, ne se résout pas avec certitude à la fin du récit. Le ton du roman, cependant, alliant biographie et discours savant, utilisant les arguments bien davantage que les outils narratifs, le rapproche de l'écrit universitaire. La seule référence à un tel texte dans le récit concerne la thèse de Nicolas, disparue : "[Nicolas] a détruit tous ses documents informatiques, disparus à jamais, incluant sa thèse, mais dont il resterait toujours une version imprimée égarée dans la bibliothèque de l'UPM » (EA : 28). Cette thèse sur Artaud, malgré une métalepse improbable, semble avoir le même statut que l'écrit biographique de Nicolas Tremblay; cela est corroboré par les quelques références à Max Brod, éditeur malgré lui des écrits de Franz Kafka. Le Texte que nous lisons, ainsi, serait un texte au statut précaire au sein de la fiction, et dont l'origine pragmatique - la thèse écrite par Nicolas Tremblay au début des années 2000 - rend tout rapprochement hypertextuel suspect. Par là, d'une ingénieuse manière, c'est le Texte, comme figure littéraire organisée, qui est frappé de négativité.

Nicolas Tremblay ne se contente pas, dans sa fiction, d'être négatif. D'ailleurs, cette négativité suffirait-elle au projet du bartleby ? Il lui faut encore se soustraire à soi-même. Sa personnalité, d'abord, s'étiole dans celle d'Artaud : " car lire la vie de Nicolas Tremblay [...] vous replongera souvent dans celle d'Artaud, comme celle d'Artaud vous ramène à plusieurs reprises à celle d'Héliogabale » (EA : 45). Puis, plus largement, dans la littérature : «au contact de la littérature [...], Nicolas sentait secrètement qu'il perdait sa personnalité » (EA : 135). Il ne lui manque plus qu’à disparaître dans le langage. C'est ce qu'il révèle dans ses notes de cours :

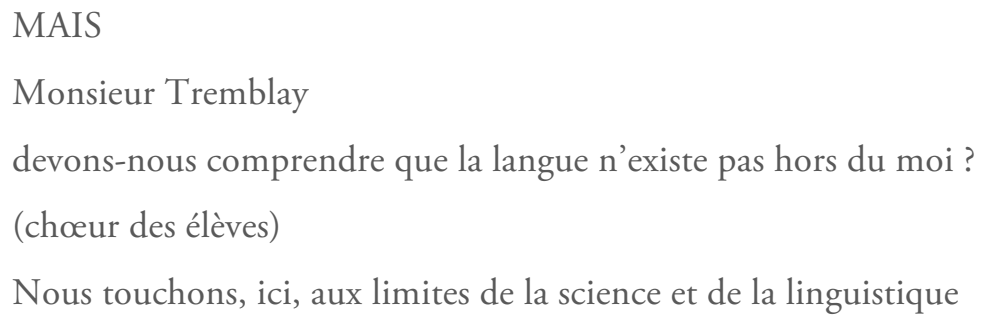




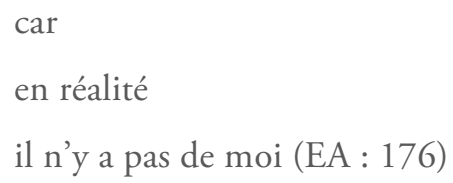

Après le Texte, l'Auteur est liquidé ; il ne s'agit pas de ne pas écrire, comme le texte ne peut, pour des raisons pragmatiques, se contenter de ne pas avoir été écrit, il lui faut ne pas être, c'est-à-dire être un non-être.

Il reste pourtant, au bout de l'expérience, le livre et il reste le vrai auteur, consubstantiel. Comme le montrait bien l'incipit de Nous autres ça compte pas, la théorie ne peut survivre à la réalité. Mais peut-être que le dernier pas n'a pas encore été franchi : suffit-il de disparaître, de se soustraire, de se confondre dans le langage pour ne pas être ? Il faudrait encore n'avoir jamais été. Jean-Nicolas, en préface, montrait son refus des origines, supposant en creux qu'il serait un tout en lui-même, sans ancêtre, sans histoire, pur ici et maintenant. C'était déjà le projet de Nicolas :

[L]'estafette se serait donc construite ou emboîtée seule, elle serait ni plus ni moins sa propre mère. De la même façon que, chez le poète, sa filiation $[\ldots]$ saute une génération, pour commencer avec les jours de la grand-mère, ici maternelle, et non avec sa période embryonnaire et foetale. En vérité, [...] [l'estafette] sortirait contre toute logique de l'anus de son aïeule [...]. Cette déformation de la réalité nie en fait la sexualité et la procréation, oppose à la matrice et à la vie, la mort, la merde. (EA : 96)

Comme Jean-Nicolas s'apprête à "renier définitivement [s]es racines ", Nicolas s'invente une origine excrémentielle, manière de contester la généalogie naturelle, manière, en même temps, d'inscrire l'engendrement, celle du Livre en l'occurrence, moins dans la création que dans la production obligatoire de déchet. Une estafette chez Artaud serait par là le déchet nécessaire d'une autogénèse littéraire négative.

\section{L'écrivain frauduleux : je n'ai pas écrit ce que j'ai écrit}

L'écrivain frauduleux s'inscrit dans la dynamique exactement inverse à celle de l'écrivain négatif. Là où le bartleby soustrayait sa présence, en effet, le fraudeur additionne. La figure de l'écrivain est alors renversée plutôt que liquidée. Tout cela s'effectue selon un rapport de dépendance entre le texte et 
son auteur, suivant la fonction-auteur foucaldienne ${ }^{4}$; Charline Pluvinet l'exprime fort clairement :

être auteur signifie être fondateur d'une ouvre, la notion d'auteur se définit nécessairement par rapport à l'existence d'un écrit qui lui confère son autorité. Une relation interdépendante unit œuvre et auteur : l'œuvre achevée consacre celui qui l'a composée comme auteur. Par conséquent, si l'œuvre fait défaut - qu'elle soit inexistante, inachevée ou qu'elle ait disparu - ce statut est alors mis en suspens et le terme se vide de son sens. (2012: 173)

Les figures du romancier sans œuvre - il prétend avoir écrit -, du romancier plagiaire ou du romancier usant de nègres s'inscrivent dans cette mouvance. Dans Document 1 de François Blais, par exemple, les narrateurs, Tess et Jude, empruntent l'identité d'un écrivain reconnu afin d'effectuer une demande de financement au Conseil des arts du Canada pour un projet de roman. Le vrai auteur du Texte - le scripteur - n'est donc pas l'écrivain, déjouant le statut auctorial et les règles de la prise de parole littéraire. Le journaliste Ivanhoé McAllister dans Catastrophes de Pierre Samson effectue quant à lui la recension d'un roman improbable "publié en 1977 à Saint-Jovite par Oblivion, une maison depuis longtemps dissoute, dans la collection Rem, lourd de 511 pages sans paragraphe, un inconnu, un inlu, un introuvable " (24). La rareté de l'œuvre, suggère cependant la narration, s'explique par son inexistence : le journaliste a créé un auteur et une œuvre pour les besoins de son travail. Le phénomène est autrement structurant dans Pleurer comme dans les films de Guillaume Corbeil.

Le titre contient déjà l'essentiel de l'enjeu du récit : il s'agit de faire semblant, et éventuellement d'ajouter un degré à ce semblant, de faire semblant comme on fait semblant dans les films. Le narrateur, un enfant hydrocéphale, raconte ainsi son rapport à sa jeune cousine, Jade, aveugle. Celle-ci ne peut aller à l'école: "Elle ne l'attendait pas vraiment, l'autobus. Elle faisait seulement semblant" (Pleurer comme dans les films [PF] : 9). Mangeant une glace, les deux enfants expriment encore par le semblant leur contentement : "Nous avons fait semblant de manger notre crème glacée avec de grandes léchées. Je m'efforçais de bien sortir la langue, pour qu'on puisse la voir se tendre, aller et venir»(PF : 13). Devant sa mère qui veut lui faire adopter une attitude tragique, calquée sur les grandes biographies d'écrivain, le narrateur ne fait pas autre chose : "Je respirais profondément et je ne savais pas pourquoi, mais il me semblait que je devais me mettre à pleurer » (PF :

${ }^{4}$ On se rappellera que, dans sa conférence «Qu'est-ce qu'un auteur ? », Foucault conçoit la "fonction-auteur ", notamment, comme un objet d'appropriation : le nom de l'auteur sert à s'approprier un discours, alors que traditionnellement un discours ne pouvait - en tant qu'acte - être possédé. 
15). Ou encore, au café, mimant l'écrivain: "Je fronçais les sourcils, contractais ma bouche et regardais à gauche et à droite, comme si j'étais en train de réfléchir" (PF : 57). La mère du narrateur, en effet, garde, dans la chambre de son fils, "toutes les biographies que l'hebdomadaire La grande revue des grands écrivains faisait paraître"( $\mathrm{PF}: 18-19)$. Cette revue au titre emphatique, construite de la gloire de la littérature, constitue la lecture privilégiée et unique du narrateur et de sa mère. Le narrateur est invité à s'inspirer de ces biographies pour modeler son attitude et devenir ainsi un grand écrivain à son tour : "Maman nettoyait cet espace de ma bibliothèque aussi souvent, en prévision du jour où ce serait ma biographie que La grande revue des grands écrivains publierait " (PF : 19). On le voit, le narrateur ne doit pas écrire de grande œuvre, il doit simplement faire semblant d'être un écrivain pour le devenir. La lecture de l'homme et l'œuvre comme la fonction-auteur de Foucault se trouvent alors singulièrement inversées. Une grande biographie, une biographie adéquate, fait l'écrivain, qu'importe l'œuvre.

Cette inversion fonde l'identité même du narrateur. Sa naissance est, en soi, livresque : "C'est un livre qui a annoncé à Maman que je deviendrais un grand écrivain et que je remporterais le prix Goncourt " (PF : 29). Après une description du livre-oracle tel l'archange Gabriel à l'annonciation, les choses paraissent se compliquer : "Puis, après avoir soulevé sa jupe, arraché sa petite culotte et être passé entre ses jambes, [le livre] a fracassé la fenêtre et est parti vers l'horizon en battant de ses deux couvertures»(PF : 29). Être de papier, littéralement, le narrateur serait fils d'un livre et d'une femme, enfanté par une œuvre et fait grand écrivain. De cette manière, son destin serait tracé, une grande téléologie marquant chacun de ses gestes :

J'ai fait une crise d'asthme ce soir-là. [...] Je toussais, je crachais, et je pouvais sentir mon visage rougir, puis bleuir. J'ai regardé en direction de ma bibliothèque et me suis dit que je ne pouvais pas mourir tout de suite, non, j'avais encore une grande œuvre romanesque à écrire. Et soudainement je me suis calmé et j'ai pu respirer normalement. (PF : 37)

Cette immortalité toute relative - le devenir détermine le présent - agit également sous la forme d'une contrainte. Confronté à plusieurs biographies, le narrateur tâche de s'y fondre: "Le soir avant de m'endormir, je regardais dans ma bibliothèque l'espace qu'occuperait ma biographie après ma mort. Je fermais les yeux et, en pensant aux photos accrochées aux murs du salon, je revoyais les différents épisodes tels que Maman me les racontait " (PF : 61). Ainsi, lorsque la mère raconte la biographie de son fils aux gens du café, ce dernier change et s'adapte, "le souvenir de tout ce qu'elle décrivait me revenait au fur et à mesure " (PF : 97). Le défi est néanmoins de taille : «Si je devais devenir un grand écrivain, il fallait que ma vie soit en tout point 
conforme à celle d'un grand écrivain. Tout ce que je disais devait être des répliques de grand écrivain. [...] Il fallait cesser d'être ce garçon qui n'était pas moi » (PF : 63). Et c'est à force de synchronie et d'imitation qu'il réussit à devenir comme un écrivain, à n'être plus un petit garçon : "Oui, l'histoire dont j'étais le personnage principal, c'était la mienne. Ça y était, j'étais moi. [...] Je m'étais devenu, enfin. Oui, je m'étais » (PF : 110). Qu'en chemin il ait écrit une œuvre semble ici indifférent.

L'œuvre, c'est effectivement là où le bât blesse dans Pleurer comme dans les films. Si être écrivain consiste à revêtir un habitus, à traverser certaines crises - le repas préféré du fils paraît changer au gré des découvertes biographiques, par exemple -, le texte, son authenticité, sa qualité, son inventivité, n'a rien à voir dans le procès identitaire. Le texte qui fonde le roman paraît à cet égard, grâce à différents indices, le fait d'intertextes multiples, carrefour de voix et de déterminismes.

L'amour enfantin et platonique d'un être difforme - le narrateur hydrocéphale - et d'une belle aveugle rappelle naturellement L'homme qui rit de Victor Hugo, Gwynplaine et Déa, le monstre et l'aveugle, dans leur amour naïf et romantique. De la même manière, une reproduction théâtrale de la fausse tentative de suicide du narrateur rappelle une scène célèbre de Hamlet, comme le ton du roman, une voix d'enfant relevant les paradoxes de la norme sociale et langagière, suggère plusieurs rapprochements avec l'écriture de Romain Gary. Si ces multiples intertextes constituent l'œuvre, la question de l'origine de celle-ci est explicitement posée au sein de l'intrigue. J'ai déjà mentionné la paternité livresque du narrateur. Il se peut que cette paternité doive se penser en termes foucaldiens : si l'auteur existe pour et par une œuvre, la question du père devient, inversement, la question de l'œuvre produite. Pour le dire autrement : quelle ouvre prodigue le statut d'auteur au narrateur ? Ne cherchons pas la réponse trop loin. Assurément, le texte en place, Pleurer comme dans les films, fait du narrateur un écrivain, et on peut lire de façon littérale son exclamation déjà citée : "Oui, l'histoire dont j'étais le personnage principal, c'était la mienne»( $\mathrm{PF}: 110)$. Le narrateur serait écrivain grâce à un récit qui le raconte écrivain. Or, une telle adéquation paraît rapide. De fait, l'exclamation du narrateur survient peu après qu'il se soit enfin mis à l'écriture. Son processus de création, cependant, laisse perplexe. Après s'être procuré un livre au Café du coin, le narrateur s'enferme dans sa chambre :

et là, derrière mon bureau, j'ai transcrit le roman dans un cahier, mot pour mot. Quand j'ai eu terminé, j'ai recommencé. [...] J'ai fait ça pendant plusieurs semaines, jusqu'à en connaître le moindre signe de ponctuation par cœur. Ce jour-là, à la tombée de la nuit, je suis sorti enterrer le livre dans le jardin, là où personne ne pourrait jamais le retrouver. [...] Le lendemain, j'ai transcrit de nouveau le roman, mais de mémoire cette fois. Il m'arrivait de me tromper et d'écrire le mauvais 
mot, un synonyme ou un terme inapproprié, et quand je relisais le texte, je le biffais et le remplaçais tout de suite. (PF : 107-108)

Le livre produit prend alors les atours du Quichotte de Pierre Ménard ${ }^{5}$; il devient une reproduction méticuleuse d'un autre texte, sauf qu'ici, cet autre invité à comparaître reste inconnu, pur symbole d'un grand roman dont le narrateur efface les traces - physiques - en suivant à la lettre ses traces écrites.

Le problème de l'énonciation demeure entier. Le narrateur est écrivain, sa vie est celle d'un écrivain, dans sa microsociété on lui en reconnaît d'ores et déjà le statut. Mais sa vie, justement, semble en même temps déterminée par plusieurs biographies d'écrivain et son origine biologique, tirée du flanc d'un livre. Plus encore, le récit qui raconte sa vie, ce récit qui constitue son œuvre, est la copie intégrale d'un autre livre. L'écrivain frauduleux ici semble donc devoir son énonciation à une fraude, une fraude au ixième degré. C'est grâce à l'addition des textes autres - intertextes, hypertextes - que cet écrivain acquiert sa voix, mais une voix inauthentique, façonnée par les autres, par les ordres de la littérature et sa doxa sacrée. La grande revue des grands écrivains, son titre, ne l'indique que trop bien : le sacre de l'écrivain, selon la formule de Paul Bénichou, est vécu dans la société de ce roman, la littérature y est une gloire qui vaut bien sa fraude. Une gloire cependant qui apparaît perdue à la fin du récit, comme le propose le destin du voisin d'en haut. Ce voisin, c'est Émile Ajar.

Le personnage d'Émile Ajar, lorsqu'il survient dans le récit, se voit aussitôt refuser tout lien familial avec le narrateur : "Ce n'est pas mon père, Émile Ajar. Il nous arrivait peut-être parfois de nous exprimer de la même façon, oui. Mais ce n'était en rien dû à un quelconque lien de parenté " (PF : 43). Sa description littéraire renvoie directement à la relation simple de l'écrivain à son œuvre: "Émile écrivait des livres, alors il ne pouvait être qu'un écrivain. Mais les écrivains, ils ne s'appellent pas Émile Ajar, non " (PF : 47). Il s'agit là de la plus grande souffrance d'Émile, qu'on lui refuse son existence littéraire en raison de son identité floue. Le désir de l'auteur, explique-t-il, est plus fort que tout: "Il m'a expliqué que lorsque les gens lisent un livre [...], ils ont besoin d'un visage, d'une image à mettre sur les mots. Sinon ils n'y arrivent pas. Ils ne voient que les caractères imprimés sur le papier $[\ldots]$ " ( $\mathrm{PF}:$ 47). Cette image, suggère-t-on, est livrée certes par le texte, mais plus encore, par une convention sociale. C'est par cette convention, raconte Émile, qu'on lui a volé son identité :

${ }^{5}$ Il s'agit du personnage de Borges mis en scène dans «Pierre Ménard, auteur du Quichotte ", nouvelle publiée dans Fictions (1944). 
quand j'ai dit que finalement j'acceptais de me présenter à la télévision, ce n'est pas moi qui y suis allé en vérité, mais quelqu'un qui avait plus la gueule d'Émile Ajar que moi. Et lui, Paul Pavlovitch [...] quand il s'est trouvé devant tous ces journalistes qui lui posaient des questions, il a fallu qu'il réponde exactement ce qu'on espérait d'Émile Ajar pour qu'ils l'écoutent. (PF : 129)

Façonnant son propre personnage pour Paul, selon les espérances et les attentes du monde littéraire, Émile en vient à n'être plus qu'un «texte de théâtre " (PF : 130), et conclut, devant le narrateur tétanisé, par ce qui ressemble à la morale du roman :

jusqu'à notre mort, nous serons toujours dans les coulisses de notre personne, à essayer de lui faire dire ce qu'elle dirait, et comme elle le dirait. [...] Nous nous efforçons de [nous] cacher en nous faisant passer pour celui que nous devrions être, en nous imitant pour tâcher de nous ressembler [...]. Mais la vérité c'est que nous sommes tous des imposteurs, les faussaires de notre propre personne. (PF : 130)

Dans les derniers moments de Pleurer comme dans les films, l'écrivain frauduleux qu'est le narrateur est confronté à l'écrivain authentique que l'on a fraudé. La propriété de la parole devient le problème principal de la fraude, puisque toute parole paraît être fraude, ce qui suggère ainsi qu'il n'y a pas d'être essentiel à exprimer. C'est le pouvoir sacré de la littérature qui se trouve alors attaqué .

Frauder la littérature, briser le lien entre l'homme et son ouvre, apparaît alors comme une dénonciation de cet ordre de discours, et aussi bien comme une libération de ses chaînes systémiques. La fraude, en ce sens, agit comme une résistance, et c'est là où l'écrivain frauduleux se situe sur le même plan que l'écrivain négatif: tous deux, à leur manière, refusent l'autorité de l'institution. Voilà, pour l'essentiel, ce qui définit «l'anti-écrivain ».

\section{L'anti-écrivain, cet écrivain en contre-jour}

L'écrivain qui n'écrit pas, l'écrivain qui n'a pas écrit son œuvre, ces phénomènes s'inscrivent au sein d'une réflexion sur le statut de l'auteur tel qu'il est représenté dans la fiction. La figure de l'anti-écrivain recoupe ces

${ }^{6}$ À cet effet, Joël Loehr et Jacques Poirier soulignent à juste titre que " pour avoir pensé la notion d'auteur à l'aune d'un modèle hors d'atteinte, la littérature s'est vouée à l'impasse. [...] Longtemps, on aura attendu de l'auteur - le poète voyant, le maître de vérité - ce qu'il ne pouvait donner ; tout comme dans la conception heideggerienne, la poésie est censée faire entendre la voix de l'Être. Une telle illusion était intenable» (2015 : 5). 
deux catégories, en suggérant moins une réflexion sur le statut de l'énonciateur majoré du titre d'" écrivain " qu'une indécision au sein du texte quant à l'origine auctoriale. Les œuvres étudiées jusqu'ici invitent à considérer ces enjeux : chez Tremblay comme chez Corbeil, le Texte est présenté sans origine, celle-ci se trouve fragilisée par le statut ambigu des personnagesécrivains. C'est dire que l'anti-écrivain est une catégorie capable d'accueillir les deux autres, ne travaillant ni dans l'addition ni dans la soustraction. L'écrivain, ici, apparaît sous la forme d'une variable ou d'une impasse.

Le phénomène peut assez bien se résumer par le titre mallarméen d'un article de Marilyn Randall: il s'agit de la "disparition élocutoire du romancier ». En guise d'étape après le " roman de l'écriture " d'André Belleau, dans lequel l'écrivain représenté accueille à la fois la réalité de l'institution et la parole littéraire, Randall avance l'idée d'un " roman de la lecture ", où " par le biais d'une intertextualité souvent exacerbée; par des commentaires sur les œuvres constitutives des mondes réel et fictif; par la lecture et le commentaire de sa propre écriture, le scripteur transformé en lecteur devient à la fois problématique et objet de son propre récit" (2006: 88-89). Ce caractère problématique, Randall l'observe dans plusieurs œuvres, grâce à la figure de la métalepse auctoriale ; ainsi, Prochain épisode de Hubert Aquin ou Le sexe des étoiles de Monique Proulx en constitue des exemples révélateurs. Robert Dion évoquait déjà, dans Le moment critique de la fiction, un " roman du critique ":

\begin{abstract}
De l'écrivain en tant qu'objet du récit, on serait donc passé, en deux décennies, à l'écrivain en tant qu'agent du récit [...]. Dans le prolongement de cette conception de l'histoire récente du roman québécois, je serais tenté d'ajouter une troisième étape, celle du romancier (ou du professeur, ou de la traductrice, ou du lecteur compétent) en situation de critique, évolution qui serait analogue à celle qui, depuis les années 1950, nous a fait passer du strict intérêt pour l'histoire (au sens de diégèse) à celui pour le discours, puis pour le métadiscours. (2009: 135-136)
\end{abstract}

Si, à la différence de Randall, Dion ne va pas jusqu'à observer une ambiguïté énonciative, sa réflexion pose néanmoins ce déplacement du scripteur dans une pensée sur le statut du texte. Le texte littéraire, propose Dion, pâlit dans la décennie 1970 face au texte du critique, en cela lié au triomphe structuraliste: "Au cours de cette décennie, la critique devient un discours hégémonique, et le professeur, qui en est le principal dépositaire, tend à monopoliser la parole légitime sur la littérature, quand il ne se fait pas luimême auteur" (2009 : 188). L'anti-écrivain devient ainsi une conséquence de la fragilité d'un statut - fragilité qu'il faut concevoir comme l'effet, d'abord, de la " mort de l'auteur " - et par la force du discours de l'autre, en littérature. J'ai ouvert cet article en citant le roman de François Blais où l'auteur reconquérait frontalement sa place unitaire. Cette reconquête laisse croire à 
une possible unité, car, comme le remarque Maurice Couturier,

ces critiques qui adhèrent toutes au dogme de la mort de l'auteur, refusent généralement de reconnaître le caractère surdéterminé du texte. [...] Dès lors que je reconnais l'existence de cette figure [d'auteur] au cœur du texte, je m'efforce par tous les moyens d'en discerner les contours et de recomposer le texte en fonction d'elle et non plus de moi, même si ma coopération est indispensable à l'émergence de cette figure. (1995: 243-244)

Lorsque dans La nuit des morts-vivants du même François Blais le récit paraît porté par des narrateurs mandatés par un auteur (absent du procès énonciatif, confiné au simple rôle de compilateur des textes, véritablement placé en contre-jour), cette unité manque. L'absence étonnante de hasards romanesques au sein du texte mine encore davantage l'autorité démiurgique de l'auteur. Lorsque l'identité de l'auteur premier du texte, par suite de métalepses, demeure problématique, l'unité surdéterminée dudit texte, comme un casse-tête qu'on n'aurait pas terminé, semble moins claire. On observe de tels phénomènes dans Conséquences lyriques de Pierre Yergeau et La revanche de l'écrivaine fantôme de David Turgeon, où l'identité auctoriale devient objet d'intrigue, mécanique en chausse-trappes, jeu de brouillage. Ce contre-jour paraît symptomatique, mais le diagnostic, par trop évident, demande quelque prudence. Si la perte de prestige ou la fin de la littérature pointe ici le nez, le caractère ludique et décomplexé de ces structures littéraires révèle peut-être autre chose.

L'hypothèse de la "disparition " ou de la "négativité " de l'auteur se vérifie dans bon nombre de récits des années 2000. Que l'auteur ne soit plus présenté dans son incapacité à écrire, mais bien dans son radical refus de la parole ou dans sa construction négative de celle-ci s'avère en ce sens révélateur. Comme l'écrit Robert Dion à propos du scripteur-critique,

pour qu'un tel recours soit possible, il faut minimalement que la pertinence sociale, culturelle et sapientielle de la littérature soit reconnue. Il faut que le texte littéraire ait acquis un certain statut, qu'il soit devenu un discours recevable non seulement dans la sphère culturelle mais dans l'ensemble de la société. (2009 : 188)

La pertinence sociale et institutionnelle du littéraire se trouve en effet confirmée par ces jeux de structures, même si elle n'est affirmée que pour, dans le même souffle, être contestée. À tout le moins, on peut dire que la société littéraire québécoise est constituée, contrairement à ce qu’observait 
Michel Biron dans son Absence du maître ${ }^{7}$ En sapant l'autorité de la parole, on en revient, suivant l'opposition de Belleau, à donner voix au code, code littéraire et moderne ; on en revient à donner toute leur force aux différentes instances et institutions. C'est là sans doute l'ultime constat auquel invitent ces écrivains en contre-jour : pour qu'on n'aperçoive que leur silhouette floue, c'est que toute l'attention doit se porter ailleurs, sur le décor dans lequel ils prennent place. L'Université, ses docteurs et professeurs, ses critiques savants et ses références ; le monde médiatique, les prix, le financement, les ventes, tout cela prend une ampleur telle dans les œuvres que la parole de l'écrivain paraît indifférente. Pour mieux dire, il semble que cette négativité n'appelle finalement qu'une chose: une communauté interprétative enfin constituée. Lorsque l'auctorialité du texte flotte dans ses pages, il invite effectivement le lecteur, le lecteur averti et capable, à en terminer le puzzle laissé en plan. Cette constitution textuelle de la communauté se fait certes contre l'institution, en l'attaquant par la fraude ou la négativité. Voilà un moindre paradoxe d'une (post)modernité littéraire sur laquelle Jacques Dubois écrivait :

En somme, ainsi décrite, la modernité n'est pas autre chose que
l'idéologie générée et exigée par l'institution autonomiste. [...] C'est
qu'à maintenir l'idée qu'il n'est d'institution que stabilisée par son code
et routinisée par sa norme, on doit convenir que le système culturel
moderne ne se reproduit qu'en cultivant ce qui le nie, c'est-à-dire la
contestation permanente du code ou encore sa perversion. (1992:137)

Le code à contester, à l'époque contemporaine, au Québec, serait cette armature littéraire, faite de médiatisations et d'organisations institutionnelles. Cela en dit plus long, sans doute, sur le code lui-même que sur ses contestataires: alors qu'au Québec des années 1950, le romancier fictif paraissait vouloir se propulser hors de sa classe d'origine - classe populaire, a fortiori - en devenant écrivain, alors que l'écrivain imaginaire voulait s'engager et parler au nom de sa nation dans les années 1960-1970, contestant les discours coloniaux et élitistes, l'écrivain d'aujourd'hui ne peut plus qu'être anti-écrivain, contestant l'ordre qui lui donne la parole. Tout se passe comme si, en fait, la littérature n'avait plus les moyens de s'opposer à quelque discours que ce soit, sinon le sien propre.

\footnotetext{
7 «Au Québec, l'écrivain paraît intégré d'emblée dans son monde, mais ce monde n'est pas le sien, comme écrivain. Il habite un monde privé de société, sa société s'entend. En termes bourdieusiens, il n'a pas de sous-champ de production restreinte où développer un réseau de relations proprement littéraires et fortement hiérarchisées"(Biron, 2000 : 37).
} 


\section{Références bibliographiques}

Belleau, A. (1999), Le romancier fictif. Essai sur la représentation de l'écrivain dans le roman québécois. Québec : Nota Bene. [1980].

Biron, M. 2000. L'absence du maître. Saint-Denys Garneau, Ferron, Ducharme. Montréal : Les Presses de l'Université de Montréal.

Blais, F. 2007. Nous autres ça compte pas. Québec: L’instant même.

Blais, F. 2011. La nuit des morts-vivants. Québec : L'instant même.

Blais, F. 2012. Document 1. Québec : L'instant même.

Bourdieu, P. 1971. "Le marché des biens symboliques ». L'année sociologique 22 : 49-126.

Corbeil, G. 2009. Pleurer comme dans les films. Montréal : Leméac.

Couturier, M. 1995. La figure de l'auteur. Paris: Seuil.

Dion, R. 2009. Le moment critique de la fiction. Les interprétations de la littérature que proposent les fictions québécoises contemporaines. Québec : Nota Bene. [1997].

Dubois, J. 1992. "L'institution du texte». Dans La politique du texte. Enjeux sociocritiques, sous la direction de J. Neefs et M.-C. Ropars. Lille : Presses universitaires de Lille : 125-144.

Foucault, M. 1994. "Qu'est-ce qu'un auteur ?". Dans Dits et écrits (1954-1988), T. I. Paris : Gallimard : 789-821. [1969].

Genette, G. 1972. «Discours du récit». Dans Figures III. Paris : Seuil : 67-273.

Lemieux-Couture, M.-C. 2012. Toutes mes solitudes! Montréal : Ta mère.

Loehr, J. et J. Poirier. 2015. "Avant-propos». Dans Retour à l'auteur, sous la direction de J. Loehr et J. Poirier. Reims : Presses universitaires de Reims : 5-11.

Marcotte, G. 1976. Le roman à l'imparfait. Essais sur le roman québécois d'aujourd'hui. Montréal : La presse.

Martel, J.-P. 2012. Comme des sentinelles. Montréal : La mèche.

Pluvinet, C. 2012. Fiction en quête d'auteur. Rennes : Presses universitaires de Rennes.

Raczymow, H. 1994. La mort du grand écrivain. Essai sur la fin de la littérature. Paris : Stock.

Randall, M. 2006. "La disparition élocutoire du romancier: du "roman de la lecture" au "roman fictif" au Québec ». Voix et images 31 (no 3) : 87-104.

Samson, P. 2007. Catastrophes. Montréal : Herbes rouges.

Tillard, P. 2011. De Bartleby aux écrivains négatifs. Une approche de la négation. Montréal: Quartanier.

Tremblay, N. 2011. Une estafette chez Artaud. Montréal : Lévesque éditeur.

Tremblay, R. 2004. L'écrivain imaginaire. Essai sur le roman québécois, 1960-1995. Montréal : $\mathrm{HMH}$

Turgeon, D. 2014. La revanche de l'écrivaine fantôme. Montréal : Le Quartanier.

Yergeau, P. 2008. Conséquences lyriques. Montréal : Québec Amérique. 